Cahiers $d u$ MONDE RUSSE

\section{Cahiers du monde russe}

Russie - Empire russe - Union soviétique et États indépendants

$50 / 4 \mid 2009$

Varia

\title{
Élisabeth Anstett, éd., Un patrimoine sous influences
}

\author{
Gábor T. Rittersporn
}

\section{OpenEdition \\ Journals}

Édition électronique

URL : https://journals.openedition.org/monderusse/7263

DOI : $10.4000 /$ monderusse. 7263

ISSN : $1777-5388$

\section{Éditeur}

Éditions de l'EHESS

\section{Édition imprimée}

Date de publication : 15 décembre 2009

Pagination : 952-955

ISBN : 978-2-7132-2261-0

ISSN : $1252-6576$

Référence électronique

Gábor T. Rittersporn, «Élisabeth Anstett, éd., Un patrimoine sous influences », Cahiers du monde russe [En ligne], 50/4 | 2009, mis en ligne le 12 janvier 2011, consulté le 03 septembre 2022. URL http://journals.openedition.org/monderusse/7263; DOI : https://doi.org/10.4000/monderusse.7263

Ce document a été généré automatiquement le 3 septembre 2022

Tous droits réservés 


\title{
Élisabeth Anstett, éd., Un patrimoine sous influences
}

\author{
Gábor T. Rittersporn
}

\section{RÉFÉRENCE}

Élisabeth ANSTETT, éd., Un patrimoine sous influences. Usages politiques, religieux et identitaires de l'image dans le monde slave. Paris : Éditions Pétra, 2009, 144 p.

1 Les usages religieux et politiques des images sont aussi anciens que les images ellesmêmes. Leurs premières fonctions connues étaient cultuelles et étroitement liées aux affaires publiques ainsi qu'à l'exercice du pouvoir. Les images pouvaient contribuer à donner un sens à l'existence de groupes et d'individus, pour autant que ceux-ci se reconnaissaient, ou refusaient de se reconnaître, dans ce qu'ils voyaient représenté. Il en allait de même - et c'est toujours le cas aujourd'hui - dans le monde slave, pour lequel l'éditrice et les auteurs de ce volume se proposent de rendre compte des emplois religieux, politiques et identitaires de diverses sortes d'images. Idéalement, le point de départ serait les images elles-mêmes, les visions qu'elles incarnent et projettent grâce à leurs dimensions symboliques.

2 Or, nous sommes loin de vivre dans un monde idéal. La plupart des chapitres de l'ouvrage ne traitent pas vraiment d'images, mais plutôt de leur support ou de leur environnement. Ceux-ci ont beau expliquer beaucoup de choses, ils n'en éloignent pas moins le regard des images en tant que telles. Si bien que les analyses auxquelles ils donnent lieu pourraient également porter sur d'autres objets, que l'on pourrait interpréter sans prêter attention à leurs particularités iconographiques. Deux chaises identiques ne font pas le même effet dans le coin d'une chambre de bonne ou sur le podium d'une salle de réception; et peu importe qu'elles aient une ornementation décorative que l'on devra déchiffrer dans les situations évoquées.

C'est à peu de chose près le problème du texte de Lubomir Hosejko. Celui-ci traite de cinéma en Ukraine postsoviétique. Cependant, les images ne l'intéressent pas du tout : en 
fait, son texte pourrait très bien s'en passer. L'auteur parle des mésaventures de metteurs en scène ukrainiens qui disposent de peu d'espace de création dans leur propre pays et exercent souvent en Russie. Il évoque également les difficultés nées des lois imposant le sous-titrage et le doublage des films étrangers dans la langue nationale. Par ailleurs, cette dernière ne facilite guère la diffusion des œuvres ukrainiennes au-delà des frontières. De plus, il semblerait que les cinéastes du pays peinent à trouver des sujets relevant de la quête d'une identité nationale. Enfin, problèmes financiers et clivages entre générations de cinéastes font que le septième art n'a pas encore trouvé la place qui pourrait lui revenir dans le paysage culturel de la nouvelle Ukraine. Le bilan est que les images n'ont pas trouvé le chemin de la pellicule et n'ont pas voix au chapitre...

Élisabeth Anstett nous fait visiter le musée d'art et d'artisanat de l'association Mémorial de Moscou. C'est une pièce étrange, qui sert également de bureau pour les conservateurs et pour le rangement des matériaux d'archives. On y expose un petit nombre d'œuvres dans des conditions faciles à imaginer : manque d'espace, éclairage défectueux, difficulté de mettre en évidence l'importance des objets individuels. C'est un peu ce que décrit l'auteur. Elle donne un aperçu des œuvres, mais surtout, comparant l'effet qu'elles produisent sur le site Internet de l'Association à l'impression qu'elles donnent au musée, elle s'interroge sur « la complexité de l'objet ou l'impossibilité d'évoquer et de mettre à distance une expérience collective dont l'écho n'est pas encore assourdi ». Élisabeth Anstett conclut, peut-être un peu rapidement, que « l'extension d'une muséographie virtuelle des camps suggère de façon ultime que ces silences construits pour les visiteurs par les muséographes [...] renvoient avant tout à la coexistence au sein de la société russe contemporaine des descendants de ces deux parties de la société soviétique (ceux qui ont fait l'expérience des camps, et ceux qui les ont envoyés dans les camps), dont l'historien et muséographe Vladimir Dukelsky nous rappelle que, depuis la fin des années 1950, elles demeurent, dos à dos en silence, soulignant aussi que "the GULAG has not vanished but has simply dissolved into Russian society, leaving behind a heavy legacy of deep rooted fear"».

On peut se demander s'il n'y avait pas entre ces deux blocs un vaste espace social à peine marqué par l'expérience concentrationnaire et si ces blocs eux-mêmes étaient aussi profondément marqués que les anciens détenus ou aussi cyniques que ceux qui les avaient persécutés. Les descendants de la génération des camps n'étaient-ils pas confrontés à des situations qui éclipsaient pour eux la mémoire collective de leurs aînés, même si celles-ci n'avaient rien de comparable avec la tragédie de la Terreur?

6 Mais, surtout, où sont les images dans cette analyse ? Car l'auteur ne s'y attarde guère. Et si par hasard elle le fait, ce n'est au prisme ni de la religion, ni de la politique, ni de l'identité. L'anthropologue analyse par exemple une élégante figurine de satin qui représente Cupidon sur la branche d'un arbre. C'est pour elle l'occasion de méditer: serait-ce l'expression de sentiments amoureux impossibles dans l'univers des camps? La fille de la même artiste, qu'une poupée représente sur le site Internet, n'est-elle pas le fruit des amours du Goulag? Méditation d'autant plus hasardeuse que nous ne connaissons de l'artiste que sa date de naissance et de décès, l'année de création de ses œuvres et leurs titres.

7 Il serait à l'évidence plus fructueux d'observer que la fille est en uniforme de soldat et que la figurine date de 1941, ce qui rend improbable sa naissance dans un camp; plus encore, de relever les affinités du Cupidon avec les putti baroques, sa pose de ballerine, son habillement pudique, la distance qui la sépare des sujets folkloriques qui dominaient 
le genre des camps, sa proximité avec certains thèmes de l'Âge d'argent de l'art russe et, en suivant ce fil d'Ariane, les rapprocher des imageries qui incluent, entre autres, l'Éros ailé décrit par Aleksandra Kollontaj et peut-être même, qui sait, les Ailes de Mihail Kuz 'min. Continuer sur cette lancée permettrait de repérer d'autres spécificités et parentés dans les motifs, dans les moyens stylistiques et dans les conventions iconographiques de la centaine d'œuvres du site et de plus de trois cents autres dans les dépôts de l'Association, de les placer dans une perspective qui ne se limiterait pas nécessairement au monde du goulag. Ce point de départ pourrait mener loin, peut-être même jusqu'à des modèles d'identification et autres sujets que faisait miroiter le volume dans l'introduction.

8 Cela valait la peine de s'attarder sur ces questions, car elles révèlent les aléas de l'entreprise. Il est difficile de parler des usages des images sans se pencher sur quelques détails de ce qu'elles représentent ni préciser comment elles le font. De ce point de vue, les icônes posent problème, puisqu'elles sont soumises à des règles canoniques qui empêchent l'auteur de s'en écarter. Le problème est bien illustré par le chapitre rédigé par Galina Valtchinova, qui porte sur les usages sociaux des images sacrées en Bulgarie et en Macédoine. Ce que dit l'auteur des légendes concernant une icône culte dans un village est bien intéressant. Elle saisit dans ces histoires les traces de conflits interreligieux et interculturels typiques des Balkans du Sud. Ceci étant dit, l'icône y apparait seulement dans sa matérialité, en tant qu'objet qui s'envole et qu'on retrouve et sur lequel on a peint la Vierge un peu par hasard. Saint Evtimij de Tarnovo pourrait faire le même office. Néanmoins, cette section de l'article est contredite par le reste du texte où il s'agit du culte de trois saintes contemporaines. Bien que suscitant une forte vénération, celles-ci ne sont pas canonisées pour autant. N'apprenant rien de leur vita, on ne comprend rien à la dévotion des fidèles. De surcroît, deux d'entre elles ont donné lieu à une production picturale dont on peut relever quelques éléments sur les illustrations qui accompagnent le texte. Malheureusement, l'auteur n'a pas jugé utile de les relier aux figures vénérées. Elle parle littéralement de "supports identitaires ", qui sont tout sauf des images et qui n'expliquent rien des identités que l'auteur voudrait dégager.

9 L'article de Tatiana Benfoughal sur la peinture populaire en Ukraine éveille quelques espoirs qui ne seront pas tout à fait déçus. Mais, une fois de plus, l'accent est mis plutôt sur un genre en général que sur ce qui reflète des modèles d'autoperception dans ces images d'une culture passablement malmenée par l'histoire. La floraison, entre le XVII ${ }^{e}$ siècle et les années 1920, d'une peinture de chevalet exécutée par des artistes du peuple et colportée sur les marchés, ainsi que son devenir à l'époque soviétique et postsoviétique, sont intéressants. Ils témoignent de l'engouement du public et de la réprobation des autorités, religieuses surtout, avant le $\mathrm{xx}^{\mathrm{e}}$ siècle, et de toutes les autorités après la révolution. Le mépris de l'Église d'Ukraine pour les icônes peintes par ces artistes n'est pas différent de celui ressenti en Russie à l'endroit de bien des bogomazy - terme d'origine ukrainienne, comme Benfoughal le dit, mais sans rappeler que chez les Russes, et ce n'est pas tout à fait par hasard, ce terme est devenu un nom de famille.

L'anthropologue a toutes les raisons de rappeler l'influence qu'exercent à partir du XIX ${ }^{e}$ siècle art académique, photographie et cartes postales. Mais, bizarrement, elle omet de relever les ressemblances entre certains tableaux ukrainiens et les lubki. Pourtant, elles sautent aux yeux sur l'une des illustrations qui mériterait d'être analysée de ce point de vue. L'image représente un cosaque, figure dont l'auteur écrit qu'elle tient le haut du pavé. Mais suffit-il de constater simplement qu'elle incarne « la revendication 
identitaire, la nostalgie des temps héroïques et l'aspiration d'indépendance ", d'autant plus que le personnage connaît une grande popularité de nos jours? D'une part, notons, par exemple, qu'il refait surface en Ukraine dans les films de l'ère soviétique sous la forme d'un cavalier rouge (parfois aussi blanc) avec une iconographie qu'il serait intéressant d'étudier. D'autre part, le cosaque de l'art populaire est loin d'apparaître toujours en guerrier vaillant, voire libérateur. Les illustrations ne montrent aucune figure vraiment martiale. Elles reproduisent des scènes galantes et, sur une œuvre du $\mathrm{XIX}^{\mathrm{e}}$ siècle, un véritable troubadour. Les dames qui décoraient la maison familiale de ces sortes d'images ne s'identifiaient peut-être pas tout à fait à des baroudeurs héroïques et il y a fort à parier que les hommes qui les achetaient avaient bien d'autres choses en tête que le combat pour l'indépendance. Examinons l'illustration, datant du XIX siècle, d'un cosaque qui demande de l'eau à une jeune personne. La composition théâtrale, les poses, le cheval blanc, l'utilisation subtile de la perspective (chose rare), la suggestion dans le lointain d'un peuplier solitaire qui pointe vers le ciel et qui, sous diverses formes, est un symbole courant de la poésie populaire, les paroles d'un chant - tout ceci pourrait dire bien des choses. Encore faudrait-il qu'on les fasse parler.

11 Paradoxalement, les sujets d'un genre pictural et la politique des mœurs qu'il motive apparaissent de façon plastique dans un chapitre qui ne cite pratiquement pas d'images. Benjamin Guichard n'avait pas accès aux images pornographiques que la censure russe interdisait au début du xxe siècle. Et pour cause : elles ne figurent guère dans les dossiers les concernant, parce que les bureaucrates vigilants les ont vraisemblablement détruites ou volées. Mais ceci ne décourage pas l'auteur, qui entreprend une fouille archéologique dans les matériaux des pièces de théâtre censurées. Judicieusement prudent, il explique que la pornographie est un concept élastique variant selon les époques et les milieux, et qui peut servir de prétexte pour simplement délégitimer des œuvres. L'historien fait le tour d'un certain nombre de thèmes et de situations que les censeurs, dans leurs rapports, jugeaient soit inacceptables, soit parfois à la limite du tolérable. Il met en évidence une imagerie et les réactions qu'elle suscitait. Pour lui, au fond, l'accusation de pornographie frappait à peu près tout ce qu'une politique moralisatrice assimilait à la culture de masse, tout autant qu'une intelligentsia peut-être libérale mais plus hypocrite qu'on ne se plaît à le croire. Beau tour de force méthodologique et belle leçon pour les apprentis iconologues.

12 Le texte où la recherche iconographique est incontestablement à l'honneur est écrit par Charles Stépanoff. L'auteur suit les métamorphoses de l'image du chaman sibérien - de la curiosité ethnographique et d'un symbole de l'autonomie revendiquée par les Sibériens jusqu'à la représentation de l'escroc sorti d'un autre âge, symbole de l'arriération et de l'exploitation du simple peuple, dangereux au point d'être assimilé à l'ennemi de classe et passé par les armes pendant les années de la Grande Terreur. L'auteur décrit et analyse des images de films documentaires et de fiction et met en évidence leurs particularités stylistiques. Il compare des films faits sur les chamans à d'autres qui mettent en scène des adversaires réels et imaginaires du pouvoir soviétique, y compris dans le domaine religieux. Stépanoff réussit à trouver et explorer des sources écrites qui approfondissent son travail d'iconologue. De plus, il déconstruit des images produites par des cinéastes qui bricolaient des reality shows dans des films prétendument documentaires. Dizga Vertov, et sa fabrique d'images tendancieuses, ne peut sortir vainqueur de la confrontation. En revanche l'anthropologue, lui, est à 
féliciter. Voilà une démonstration des procédés et des possibilités de l'iconologie que le volume n'exploite pas vraiment. 